\title{
Medical decision-making errors due to faulty heuristics in the pediatric emergency department and the use of mindfulness
}

\begin{abstract}
Unexplained variation in medical decision-making continues to be associated with significant medical errors in the United States. These decisions result from a complex interplay of rapid inductive processes known as heuristics and more methodical deductive processes. Unfortunately, in the pediatric emergency department, the speed and complexity of problems require extensive dependence on heuristics which are subject to negative influences such as biases, prejudices, and opinions. Eight fictional patient vignettes describing potentially biased decision making are described. Through the use of mindfulness, health care providers may regain some control over these involuntary processes and thereby improve patient care.
\end{abstract}

Volume 7 Issue 4 - 2017

\author{
Ron DWaldrop \\ Department of Pediatric Emergency Medicine, Inova Loudoun \\ Hospital, USA \\ Correspondence: Ron D.Waldrop MD, Department of \\ Pediatric Emergency Medicine, Inova Loudoun Hospital, \\ Leesburg,Virginia, USA, Tel 225-247-2992. \\ Email dellmd120499@gmail.com
}

Received: November 21, 2017 | Published: December 27 2017

\section{Introduction}

In 1999, the Institute of Medicine published the first in a series of ongoing reports on the American medical system. ${ }^{1}$ The goals of these reports were in part to describe the role of medical errors in patient clinical outcomes and excess healthcare cost. Initiatives proposed included institution of electronic health records, standardization of high risk encounters, medical error initiatives, improved reporting practices, and institution of facility safety practices. ${ }^{2}$ Gains have been made in decreasing medical errors since this initial report nonetheless, many obstacles still exist to decreasing medical errors including lack of transparency between electronic health records, lack of agreement and implementation of clinical practice guidelines based on evidence based practices, weaknesses in the discharge process from encounters, and lastly as well as possibly most importantly unexplained variation in physician medical decision-making practices. ${ }^{3-5}$

To address these variations, healthcare providers must become more acutely aware of both conscious and rapid unconscious decision-making processes known as heuristics especially in a busy pediatric emergency department. Similarly, they must have more awareness and control over personal biases prejudices, and opinions which may negatively influence these heuristics. In order to do so, the introduction of better mindfulness into daily practice may be necessary. Below are eight patient fictional vignettes which describe real world pediatric emergency department events which may have been negatively affected by the use of faulty heuristics. This is followed by a discussion of cognitive processes involved in decisionmaking, the influence of biases, prejudices, and opinions as well as the role of mindfulness in improving clarity of decision-making.

\section{Case vignettes}

\section{Case I}

An intoxicated 18-year-old male arrived late one night to the pediatric emergency department (PED) with mid-sternal chest pain. The patient is argumentative and uncooperative thus, alienating the staff. The staff is familiar with this patient from previous visits and desires to discharge him as quickly as possible.

Workup after a brief examination included electrocardiogram with nonspecific ST changes and chest $\mathrm{x}$-ray which were both interpreted as normal. The patient was rapidly discharged to the care of friends with the diagnosis of pleurisy and no specific follow up or discharge instructions are given. The patient returned 2 hours later and subsequently is diagnosed with cocaine induced myocardial infarction with significant cardiac injury.

\section{Cases 2-4}

Three different teenage females presented to the PED via triage with anxiety and agitation according to family members. Each patient sees a therapist for psychological issues. They all have a perceived negative attitude toward staff members and uncooperative. The staff wished to discharge these uncooperative teenagers as soon as possible because they are "just being dramatic". Workup consisted of only rebreathing into a brown paper bag which calmed each somewhat. All three were diagnosed with anxiety associated with hyperventilation and were discharged with optional follow up with their primary care provider and therapist. All 3 returned within the next 24 hours and were subsequently diagnosed with pulmonary embolism, hyperthyroidism, and epiglottitis in an incompletely immunized immigrant respectively.

\section{Case 5}

An 8-year-old male of a particular nationality (your choice) presents to the PED with complaints of headache, being argumentative, stumbling, and having behavior outbursts. Due to the staff experience with this particular nationality it is assumed this child's actions are the result of poor parenting and typical demeanor. After a cursory examination, the child is discharged with the diagnosis of behavioral problem and instructed with return to their primary physician for counselling. The child returned with the family within 24 hours and is subsequently diagnosed with a brain tumor and early hydrocephalus.

\section{Case 6}

A 15-year-old female with sickle cell disease presented to the PED with abdominal pain for 12 hours which she describes as different than her usual pain. She is well known to the staff and is known for requiring multiple doses of opioid pain medication and is rumored to have drug-seeking behaviors. Blood studies were baseline for her and multiple doses of pain medications were given as well as a prescription for opioids at home. She was discharged with the diagnoses of sickle 
cell disease with pain crisis and drug-seeking behavior to follow up. She returned with 24 hours and a more in depth workup reveal hematuria and ultrasound demonstrated portal vein thrombosis.

\section{Case 7 and 8}

A 54-year-old male with substernal chest pain and a 3-week-old female with $103 \mathrm{~F}$ fever presented simultaneously to an ED. Three hours later the hospital proudly displays the man's ST segment elevation myocardial infarction being managed in the cardiac catheterization lab within 65 minutes. Unfortunately, the 3-week-old infant waited 2 hours for management. The infant was subsequently diagnosed with bacterial meningitis and has permanent brain injury for which there was no celebration.

\section{Discussion}

Medical decision making may be divided into 2 categories: inductive and deductive reasoning. ${ }^{6}$ Inductive reasoning also known as type 1 data or intuitive reasoning consists of rapidly accessed information and is dependent on effective communication skills. Often intuitive decisions are made based on apparent information available without further depth of thought. Inductive decisions are heavily influenced by an individual's metacognition which is defined as one's personal collection of rapid innate cognitive processes (heuristics). These processes are heavily influenced by consciously and unconsciously determined prejudices, opinions, biases, and past experiences and collectively results in gestalt thinking. ${ }^{7}$ Gestalt thinking may be defined in medical decision making as believing "One's first impression is usually right". Another historical variation of this medical decision making adage is referred to as "Occam's Razor" and may be stated as "When you hear hoof beats think horses not Zebras". 8

Conversely, deductive or type 2 data refers to methodical cognitive access of information accumulated as a result of didactic and technical training with subsequent testing and application as well as implementation of algorithmic thinking. ${ }^{6}$ Early applications of deductive reasoning included the development and use of the scientific method and the proposed use of extensive differential diagnoses in psychiatric decision making by Kraepelin. ${ }^{9,10}$ Informally, it is also taught in medical training that words such as always, never, every, are too absolute and all will inevitably lead to some diagnostic failures without further consideration. Indeed, the modern application of evidence based medicine in medical decision making is an attempt to tilt the process as far toward consistent deductive reasoning as possible.

Nonetheless, there must be a balance between inductive and deductive reasoning in order to create a complete picture of a patients mental, physical, and social condition. To quote Albert Einstein: "The intuitive mind is a sacred gift and the rational mind is a faithful servant. We have created a society that honors the servant and has forgotten the gift".

The PED presents an environment requiring rapid access to information for medical decision making and potential excessive dependence on faulty involuntary heuristics. As Reason described it "the place where flesh and blood decision making occur...real decision making on the frontline when resources are scarce, time constraints apply, and shortcuts are sought". "Croskerry is his sentinel article specifically addressing medical decision making in emergency departments said it best: "emergency medicine as a disciple has been described as a natural laboratory of error. In this milieu, decision making is often naked and raw with its flaws highly visible. Nowhere in medicine is rationality more bounded by relatively poor access to information and limited time to process it, all within a milieu renown for it error producing conditions. It is where heuristics dominate and without them emergency departments...would grind to a halt. It is also where heuristics have been seen to catastrophically fail. ${ }^{12}$ Hence, rapid, efficient, cost conscious, and patient sensitive accurate decision making in the PED requires the use of fine-tuned inductive reasoning in combination with thorough, well thought out, sound deductive reasoning.

Elements of medical diagnostic error can be separated into deductive and inductive causes. ${ }^{6}$ Deductive errors may be actively improved by improvement in knowledge and procedural training as well as training in algorithmic thinking. Conversely, inductive reasoning errors are associated with metacognition such as the poor communication skills, poor heuristics with preconceived ideas, overconfidence, conscious and unconscious biases, and overt deep seeded prejudices and stereotyping. Taken together, this can be described in modern language as a lack of mindfulness..$^{13} \mathrm{~A}$ lack of mindfulness can also be described as failure to attend to subtle cues during a patient encounter which suggest uncertainty or cognitive dissonance regarding the diagnosis. ${ }^{14}$ Cognitive dissonance is a theory of human motivation that asserts that it is psychologically uncomfortable when everything does not make sense. Cognitive dissonance, being unpleasant, motivates a person to change his cognition, attitude, or behavior. In our situation, cognitive dissonance should directly affect medical decision making if acknowledged. Poor heuristics may obscure cognitive dissonance needed to make a more accurate assessment.

The number of potential biases in the practice of emergency medicine are myriad and more common ones are listed in Table 1. Many of these influences can be envisioned in the case scenarios presented. One of the most important biases is anchoring. Anchoring may occur in many forms ranging from triage labelling by personnel at the front desk, to diagnostic labelling due to frequent visits for similar complaints, and finally to previous diagnostic impressions either by the specific provider during previous encounters or by previous consultants. It is much simpler and less taxing to assume the perceived or previous diagnosis and proceed accordingly, rather than rethinking the presentation and possibilities while maintaining a wide differential diagnosis. For example, when does the patient with chronic constipation really need to have appendicitis considered? In addition, typical distractions in emergency medicine may exacerbate the use of faulty heuristics in medical decision making including stress, cognitive fatigue, high intensity decision making, phone interruptions, financial and reimbursement pressures as well as dependence or requirement to adhere to clinical practice guidelines without consideration of the patient's individual circumstances. In the name of simplifying decision making, it is forgotten that clinical practice guidelines are merely considerations and not formally considered the standard of care and are treated as such in the legal system. They are not intended to alter individualized medical decision making base on a patient's specific circumstance.

Affective error Tendency to convince one's self what you wish to be true is actually true.

\section{"Hope it is a migraine instead of subarachnoid hemorrhage"}

Ambiguity Effect Tendency to only test for high probability illness instead of those with less or unknown probability.

"Influenza versus Chikungunya fever in traveler"

Anchoring Tendency to settle on a final diagnosis based on a few facts or opinions and not allowing conflicting data to affect 
decisions. Closely related to Diagnostic Momentum which is the tendency to accept a diagnosis without question in the face of other probabilities. Triage Bias is a common manifestation

"The triage nurse it's just strep throat"

"The patient has a previous diagnosis of?"

"The patient has been seen here three times for?"

Ascertainment Bias Tendency to see what you expect to see based on patient characteristics and complaint. May contain elements of stereotyping including culture, age, and gender bias.

"Those (nationality, culture of choice) coddles their kids and let them be dramatic"

Availability Bias Belief that common diagnoses are common and rare diagnoses are rare so the odds are in my favor if I do not make that rare diagnosis. Related torecent case bias or significant case bias as well as gambler's bias. Also, related to posterior probability bias which assumes patients with certain symptoms will have the same diagnosis as other patients recently.

"Meningitis is unlike to be the cause of his neck pain so let's disregard the diagnosis"

"Right lower quadrant pain must be our third appendicitis today"

Belief Bias Tendency to accept diagnoses or treatments regardless of evidence based data.

\section{"TPA should be used in all hemiplegic strokes"}

Blind Spot Bias Tendency to fail to recognize or acknowledge personal weaknesses or potential cognitive errors. Related to Dunning-Kruger Effect where unskilled providers underestimate their ability and skilled providers tend to overestimate their abilities. These lead to both errors of commission (tendency toward action) and omission (tendency toward inaction)

"I know appendicitis when I see it...Let's operate"

Confirmation Bias Tendency to utilize evidence which supports initial opinion and ignore evidence which contradicts it.

Disconfirmation is an essential check to govern final diagnostic impressions.

"The vomiting is due to pneumonia so let's just do a chest $x$-ray and prove it"

Framing Bias Diagnostic decisions are governed by the environment in which the patient is seen.

"Primary care patients are not as sick as emergency department patients so extensive workup is not warranted"

Fundamental Attribution Bias Tendency to blame a patient for their illness based on personality or some characteristic.

\section{"He is a narcissist so his symptoms are just in his head"}

Representative Bias Tendency to use Occam's Razor in rationalizing a diagnosis. Assuming typical presentations will result in typical diagnoses. Related to Zebra Retreat in order to avoid overzealous diagnostic effort.

"Unilateral tonsillitis is just strep so just treat it with antibiotics"

Yin Yang Bias Tendency to believe a patient cannot be significantly ill despite symptoms due to multiple negative tests. May also arise from over-reliance on previous negative test to assign too low of a pre-test probability. May result from failing to acknowledge a false negative rate in testing.

"Cannot be really sick since the patient has already had an extensive work-up"

System Bias Orientation of a care system toward efficiency, reimbursement, comfort level, and resources to the point of neglecting diagnoses outside its interest.

"Surgical areas make more money so let's update that area instead of other less profitable areas"

Table I Common biases in medical decision making potentially resulting in errors in the patient care setting

Common heuristic errors include using representative heuristics ("all patients who look like this have this"), anchoring either through caregiver chain or previous experience, and use of "strong but wrong rules "not supported by evidence based medicine such as "all right lower quadrant pain represents appendicitis". Similarly, deep seated prejudices may alter perception such as those based on gender, past history, political affiliation, culture, age, occupation, and countertransference (transfer of involuntary feelings on to a patient based on personal experiences of caregiver) previous patients.

The 8 case vignettes describe real world encounters which occur in the PED every day. Without delving into the specifics of each scenario, each case illustrates the potential for faulty heuristics to negatively influence metacognition and intuitive medical decision making. Case 1 is an example where medical providers may have a complete distaste for managing intoxicated uncooperative individuals. Biases such as age stereotyping ("heart issues do not occur at this age"), time constraints (busy ED), past medical history of the same presentation (anchoring), and diagnostic momentum based on the collective negative attitude of the entire department toward this patient may lead to a rapid, intuitive, incorrect, and avoidable medical errors.

Similarly, the 3 patients in cases 2-4 may have elicited negative feelings resulting from impatience with young perceived "dramatic" female patients. Focusing on the psychosocial dysfunction rather than physiologic determinants may have led to a delayed diagnosis of these 3 life threatening conditions. Case 5 illustrates potential cultural (perceived parenting practices) and age bias in the management of child with altered behavior (unruly versus altered mental status) with subsequent delay in the diagnosis of a catastrophic brain tumor. Case 6 illustrates numerous potential biases leading to delayed diagnosis including cultural, anchoring to previous diagnoses, countertransference ("all those patients are alike") and stereotyping drug seeking behavior versus illness requiring pain management. Clearly, better listening may have discerned a change in pattern of pain and altered diagnostic course. Finally, cases 7 and 8 illustrate potential forms of system biases including preference toward easily diagnosed adult patient with a high visibility and reward, financially biases due to reimbursement, and overall department preparation and comfort issues.

Recently, improved mindfulness has been proposed as a method to improved medical decision making. ${ }^{13}$ (Table 2) Mindfulness refers to "being completely present with the patient through training, insight, and experience using constant self-monitoring and self-reflection". This requires accurate eliciting information in a nonjudgmental compassionate manner after achieving good rapport through good listening skills. Subsequently more accurate decisions may be made in concert with critical curiosity using evidence based medicine practices 
but individualized to the specific patient circumstances. Finally, the uncertainties of medical practice should be recognized and voiced within full patient disclosure in order to avoid fixed impressions without a definite deductive basis.

Table 2 Elements of mindfulness

Being present with the patient through insight and experience
Building rapport and listening
Critical curiosity
Algorithmic problem solving attitude
Effectively obtaining and transmitting information
Evidence based decision making
Acknowledgement of uncertainty
Maintaining competent technical skills
Nonjudgmental (conscious or unconscious)
Compassionate advocate
Self-monitored and self-reflective

Table 3 Guarding against bias in medical decision making

Train metacognition by discovering personal biases, opinions, and prejudices Use reflection and simulation to train decision making

Recognize use inductive heuristics versus deductive reasoning in personal decision making

Develop interpersonal communication skills especially listening

Use critical curiosity and modern memory assistants to generate differential diagnosis

Recognize cognitive dissonance and institute diagnostic pauses

Freely acknowledge diagnostic uncertainty

Despite major advances in the practice of medicine, there have been many factors leading to the decay of mindfulness in medical decision making including decline of the personal physician mindset and loss of a medical home, emphasis on elimination of variation of care, increased financial and documentation burdens, and time constraints. With a renewed interest in mindfulness in medical decision making, many benefits have been described including provider growth and satisfaction, care individualization as well as improved patient satisfaction and outcomes. True mindfulness requires extensive debiasing of personal metacognition and its influences through active reflection and feedback.

Guarding against bias in medical decision making first requires a commitment to understand your personal biases arising from metacognition through reflection and simulation (Table 3). Know your tendencies and within reason attempt to leave your personal beliefs at home. Develop excellent interpersonal communication skills especially listening. Maintain critical curiosity for each individual encounter and freely assess information through 21 st century memory assistants to access lists, explanations, and algorithms to generate a wide and inclusive differential. Learn to recognize cognitive dissonance and respond to it with a diagnostic pause and review. Finally, freely acknowledge medical uncertainty with all those involved and allow new information to guide diagnostic detours toward optimal decision making.

\section{Acknowledgements}

None.

\section{Conflicts of interest}

None.

\section{Funding}

None.

\section{References}

1. Kohn LT, Corrigan JM, Donaldson MS. (Institute of Medicine) To Err is human: building a safer health system. Washington DC; National Academy Press, USA. 1999.

2. Committee of Quality of Health Care in America and the Institute of Medicine. Crossing the quality chiasm: A new health system for the $21 \mathrm{st}$ Century. Washington DC: National Academy press, USA. 1999

3. Krumholts HM. Variations in health care, patient preferences, and high quality decision making. JAMA. 2013;10(2):151-152.

4. Classen DC, Pestotnik SL, Evans RS, et al. Computerized surveillance of adverse drug events in hospitalized patients. JAMA. 1991;266(20):2847-2851.

5. Martin AM, Michael Daniel. Medical errors-the third leading cause of death in the US. BMJ. 2006. p.353.

6. Saposnik G, Donald R, Christian CR, et al. Cognitive biases associated with medical decisions: a systematic review. BMC Med Inform Decis Mak. 2016;16:138-154.

7. Cervelli G, Borghi L, Lippi G. Do clinicians decide relying on primarily on Bayesian principles or on Gestalt perception? Some pearls and pitfalls of Gestalt perception in medicine. Intern Emerg Med. 2014;9(5):513-519.

8. Groopman J. How Doctors Think. Houghton Mifflin. 2007;117(10):2738

9. Achinstein P. to Science Rules: A Historical Introduction to Scientific Methods. Johns Hopkins University Press, USA. 2004. p.1-5.

10. Krapaelin E, DiefendorfAR. Clinical Psychiatry; a textbook for students and physicians. The Macmillan, USA. 1904.

11. Reason J. Human Error, Cambridge University Press, USA. 1990.

12. Croskerry P. The importance of cognitive errors in diagnosis and strategies to minimize them. Acad Med. 2003;78(8):775-780

13. Ludwig DS, Kabat-Zinn J. Mindfulness in Medicine. JAMA. 2004;300(11):1350-1352.

14. Festinger L. A theory of cognitive dissonance. Stanford University Text, USA. 1962. 